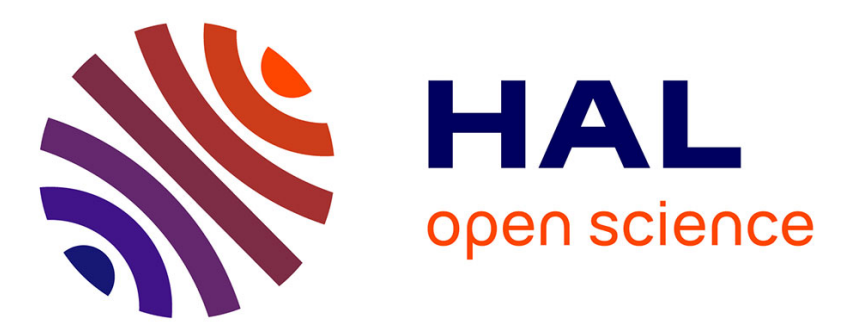

\title{
On the generation of a reverse Von Karman street for the controlled cylinder wake in the laminar regime
}

\author{
Michel Bergmann, Laurent Cordier, Jean-Pierre Brancher
}

\section{To cite this version:}

Michel Bergmann, Laurent Cordier, Jean-Pierre Brancher. On the generation of a reverse Von Karman street for the controlled cylinder wake in the laminar regime. Physics of Fluids, 2006, 18 (02), pp.028101. 10.1063/1.2171193 . inria-00338843

\section{HAL Id: inria-00338843 \\ https://hal.inria.fr/inria-00338843}

Submitted on 14 Nov 2008

HAL is a multi-disciplinary open access archive for the deposit and dissemination of scientific research documents, whether they are published or not. The documents may come from teaching and research institutions in France or abroad, or from public or private research centers.
L'archive ouverte pluridisciplinaire HAL, est destinée au dépôt et à la diffusion de documents scientifiques de niveau recherche, publiés ou non, émanant des établissements d'enseignement et de recherche français ou étrangers, des laboratoires publics ou privés. 
On the generation of a reverse Von Kármán street for the controlled cylinder wake in the laminar regime

Michel Bergmann, ${ }^{*}$ Laurent Cordier, and Jean-Pierre Brancher

$$
\begin{aligned}
& \text { LEMTA, UMR } 7563 \text { (CNRS - INPL - UHP) } \\
& \text { ENSEM - 2, avenue de la forêt de Haye } \\
& \text { BP } 160 \text { - } 54504 \text { Vandœuvre cedex, France }
\end{aligned}
$$

(Dated: December 8, 2005) 


\begin{abstract}
In this Brief Communication we are interested in the maximum mean drag reduction that can be achieved under rotary sinusoidal control for the circular cylinder wake in the laminar regime. For a Reynolds number equal to 200, we give numerical evidence that partial control restricted to an upstream part of the cylinder surface may increase considerably the effectiveness of the control. Indeed, a maximum value of relative mean drag reduction equal to $30 \%$ is obtained when applying a specific sinusoidal control to the whole cylinder, where up to $75 \%$ of reduction can be obtained when the same control law is applied only to a well selected upstream part of the cylinder. This result suggests that a mean flow correction field with negative drag is observable for this controlled flow configuration. The significant thrust force that is locally generated in the near wake corresponds to a reverse Kármán vortex street as commonly observed in fish-like locomotion or flapping wing flight. Finally, the energetic efficiency of the control is quantified by examining the Power Saving Ratio: it is shown that our approach is energetically inefficient. However, it is also demonstrated that for this control scheme the improvement of the effectiveness goes generally with an improvement of the efficiency.
\end{abstract}

Keywords: Partial rotary control ; Cylinder wake ; Drag minimization ; reverse Kármán vortex street. 
The control of the cylinder wake flow by sinusoidal rotation (Fig. 1) was intensively studied numerically ${ }^{1-3}$ and experimentally ${ }^{4}$. Generally, a global rotation of the cylinder is considered, i.e. the whole cylinder oscillates around its principal axis. When the wake flow is controlled by rotary oscillation, a maximum value of relative mean drag reduction of about $30 \%$ could be obtained ${ }^{5,6}$ for a Reynolds number equal to 200. In Ref. 7, Protas and Wesfreid argued that this mean drag reduction seems to be associated with control driving the mean flow toward the unstable symmetric state i.e. the basic flow. Therefore, as it was already pointed out by Protas and Wesfreid, is it possible for the cylinder wake flow to obtain under periodic forcing, a mean drag reduction lower than in the basic flow? The aim of this Brief Communication is to demonstrate numerically that a more effective drag reduction can be achieved by controlling only a limited part of the cylinder surface ${ }^{8}$. In our approach the cylinder boundary is divided into two regions: an upstream part on which a specific control law discussed below is applied, and the remainder of the cylinder which is not controlled. Hereafter, the upstream controlled region is geometrically defined by $-\theta_{c}<\theta<+\theta_{c}$ (see Fig. 1) where $\theta$ and $\theta_{c}$ are respectively the curvilinear coordinate of a point on $\Gamma_{c}$ and the control angle. By convention, these angles are initialized at the front stagnation point of the cylinder. Obviously the case $\theta_{c}=180^{\circ}$ corresponds to the rotation of the cylinder as a whole. The objective of this numerical experience is to determine the value of $\theta_{c}$ such that the reduction of the mean drag coefficient is maximized.

Here, the incompressible and viscous flow around a circular cylinder of diameter $D$ (radius $R$ ) is considered in a two-dimensional domain $\Omega$ (Fig. 1). The Reynolds number $R e=$ $U_{\infty} D / \nu$ is equal to 200 , where $U_{\infty}$ is the uniform velocity of the incoming flow and $\nu$ is the kinematic viscosity of the fluid. The cylinder is animated with an unsteady tangential velocity $V_{T}$, possibly equal to zero for the uncontrolled part of the cylinder surface. The 
Navier-Stokes equations are solved using a fractional step method in time and a finite element method in space (see Ref. 5).

Let $u$ be the velocity component in the $x$-direction, $p$ be the pressure and $\boldsymbol{n}$ be the external normal vector to the boundary $\Gamma_{c}$ of the cylinder, the drag coefficient is given by:

$$
C_{D}(u, p)=\int_{\Gamma_{c}} 2 p n_{x} d \Gamma-\int_{\Gamma_{c}} \frac{2}{R e}\left(\frac{\partial u}{\partial x} n_{x}+\frac{\partial u}{\partial y} n_{y}\right) d \Gamma
$$

where all the variables are non-dimensionalized with respect to the cylinder diameter $D$ and the oncoming flow $U_{\infty}$. For a circular cylinder, the mean temporal drag coefficient estimated over a finite horizon $T$ writes:

$$
\left\langle C_{D}\right\rangle_{T}=\int_{0}^{2 \pi} \underbrace{\frac{1}{T} \int_{0}^{T} 2 p n_{x} d t R}_{\mathcal{C}_{D}^{p}(\theta)} d \theta+\int_{0}^{2 \pi} \underbrace{\frac{1}{T} \int_{0}^{T}-\frac{2}{R e}\left(\frac{\partial u}{\partial x} n_{x}+\frac{\partial u}{\partial y} n_{y}\right) d t}_{\mathcal{C}_{D}^{\nu}(\theta)} R d \theta .
$$

In a first stage, the cylinder is considered to rotate as a whole $\left(\theta_{c}=180^{\circ}\right)$ with a non dimensional sinusoidal velocity $\gamma(t)=V_{T}(t) / U_{\infty}=A \sin \left(2 \pi S t_{f} t\right)$. Hereafter, the parameters $A$ and $S t_{f}$ are selected as the values which minimize the mean drag coefficient of the wake flow. In Ref. 5, a series of simulations with various amplitudes $A$ varying from 0 to 6.5 per step of 0.5 and various forcing Strouhal numbers $S t_{f}$ varying from 0 to 1 per step of 0.1 was carried out. The numerical results of this open loop control approach demonstrated (see Fig. 31 in Ref. 5) that the mean drag coefficient was minimized for a control law $\gamma_{\text {min }}$ characterized by the parameters $\left(A_{\text {min }}, S t_{f_{m i n}}\right)=(4.3,0.74)$. The corresponding relative mean drag reduction amounts to more than $30 \%\left(\left\langle C_{D}\right\rangle_{T}\right.$ varies from 1.39 to 0.99$)$. By comparison, the mean drag associated to the basic flow is 0.94. Therefore, in this case, as it was previously discussed in Ref. 7, the lower limit of the mean drag coefficient, even for a controlled flow, seems to be the drag associated with the basic flow. The wake flow generated using the control law $\gamma_{\min }$ is symmetric and tends to the wake associated with the 
unstable stationary basic flow (see Ref. 5). Moreover, the controlled flow exhibits a vortex street thinner than the one obtained for the uncontrolled flow.

Now, it is of interest to examine the relative contributions of the different quantities $\mathcal{C}_{D}^{p}$ and $\mathcal{C}_{D}^{\nu}$ to $\mathcal{C}_{D}=\mathcal{C}_{D}^{p}+\mathcal{C}_{D}^{\nu}$ (see Eq. (1)) with respect to the angle $\theta$. Such representations are done in Fig. 2 for the uncontrolled flow and in Fig. 3 for the global controlled flow with $\gamma_{\min }$. The light grayed and dark grayed areas correspond to the angles for which the fluid exerts on the cylinder a drag force and a thrust force respectively. It is noticeable (compare Figs. 2 and 3) that the application of the control law $\gamma_{\min }$ is effective only on the upstream part of the cylinder. Then, it is natural to expect that the effectiveness of the control law $\gamma_{\text {min }}$ increases as a controlled upstream part of the cylinder is coupled to an uncontrolled downstream part. Different numerical experiences have been successively considered for $\theta_{c}$ varying from $0^{\circ}$ (uncontrolled flow) to $180^{\circ}$ (global controlled flow) by step equal to $30^{\circ}$. In Fig. 4 , we notice that for angles $\theta_{c}$ contained between $73^{\circ}$ and $177^{\circ}$, the mean drag coefficient is lower than the minimal expected value corresponding to the basic flow ${ }^{7}$, suggesting that a mean flow correction field with negative drag can be obtained for this controlled configuration. Moreover, the maximal mean drag reduction is obtained for $\theta_{c}=120^{\circ}$. Figure 5 is equivalent to Fig. 3 but corresponds to the case where the rotary partial control characterized by $\theta_{c}=120^{\circ}$ is considered. Figure 5 clearly demonstrates that, for this particular control, almost all the surface cylinder contributes to the relative reduction of the term $\mathcal{C}_{D}$. In comparison with Fig. 3, it can be inferred that the major improvement is obtained near the rear stagnation point. In consequence, as we can see in Figs. 4 and 6, the mean drag coefficient is then greatly reduced. Indeed, this coefficient decreases to a value equal to 0.34 corresponding to a relative mean drag reduction greater than $75 \%$. Moreover, the drag coefficient becomes periodically negative expressing the generation of a propulsive effect (see 
Fig. 6). However, whatever the value of $\theta_{c}$, the control is not energetically efficient. The Power Saving Ratio (PSR) as defined in Ref. 7 is everywhere less than unity (see table I). In spite of that, it is remarkable that as soon as $\theta_{c}$ is greater than $30^{\circ}$, the efficiency is improved compared to the case where $\theta_{c}=180^{\circ}$ demonstrating, that for $\theta_{c}$ contained between $73^{\circ}$ and $177^{\circ}$, the improvement of the effectiveness goes with an improvement of the efficiency.

Next in Fig. $7(a)$ the organization of the vorticity $\omega_{z}$ in the near wake is presented. Alternatively, positive (resp. negative) vortices are shed in a region where the net mean vorticity is of opposite sign. This phenomenon is physically similar to the one observed in fish-like locomotion ${ }^{9}$ or flapping wing flight ${ }^{10}$. The upstream surface cylinder produces thrust as it oscillates developing a reverse Kármán vortex street that corresponds, at least locally, to a jet-like average vorticity profile. The moving walls on a part of the cylinder surface have the effect of increasing the flow momentum that may be large enough to overcome the adverse pressure gradient formed at the rear part of the cylinder and to delay the separation, resulting in the pressure drag reduction (see Fig. 4). As shown in Fig. 7(b), the Kármán vortex street tends to disappear in the far wake. Moreover, the wake flow is quasi-symmetric and its width is greatly reduced in comparison with the global controlled case (see Ref. 5).

In this Brief Communication, we demonstrated numerically that the effectiveness of a partial control restricted to an upstream part of the cylinder surface may be significantly higher that the one corresponding to the rotation as a whole. Indeed, while a relative mean drag reduction of about $30 \%$ is obtained when the control $\gamma_{\min }$ is applied on the whole cylinder, this reduction becomes greater than $75 \%$ when a partial control characterized geometrically by $\theta_{c}=120^{\circ}$ is considered. In this case, the value of the mean drag coefficient is even lower than that obtained for the basic flow, suggesting that a mean flow correction field with negative drag is observable. Physically, a partial control gives rise in the near 
wake to a reverse Von Kármán street that generates locally a significant thrust force. This unsteady flow control mechanism is similar to the drag reduction phenomenon encountered in fish-like locomotion where fish and cetaceans employ instinctively their flapping tails and several fins to produce propulsive and maneuvering forces.

* Electronic address: Michel.Bergmann@ensem.inpl-nancy.fr

1 J.-W. He, R. Glowinski, R. Metcalfe, A. Nordlander, and J. Périaux, "Active control and drag optimization for flow past a circular cylinder. Part 1. Oscillatory cylinder rotation," J. Comp. Phys., 163, 83 (2000).

${ }^{2}$ C. Homescu, I. Navon, and Z. Li, "Suppression of vortex shedding for flow around a circular cylinder using optimal control," Int. J. Numer. Meth. Fluids, 38, 43 (2002).

3 B. Protas and A. Styczek, "Optimal rotary control of the cylinder wake in the laminar regime," Phys. Fluids, 14, 2073 (2002).

4 P. Tokumaru and P. Dimotakis, "Rotary oscillatory control of a cylinder wake," J. Fluid Mech., 224, 77 (1991).

5 M. Bergmann, L. Cordier, and J.-P. Brancher, "Optimal rotary control of the cylinder wake using proper orthogonal decomposition reduced order model," Phys. Fluids, 17 (2005).

6 M. Bergmann, L. Cordier, and J.-P. Brancher, "Control of the cylinder wake in the laminar regime by Trust-Region methods and POD Reduced Order Models," $44^{\text {th }}$ IEEE Conference on Decision and Control and European Control Conference, 12 - 15 December 2005, Seville, Spain.

7 B. Protas and J. Wesfreid, "Drag force in the open-loop control of the cylinder wake in the laminar regime," Phys. Fluids, 14, 810 (2002).

8 B. Choi and H. Choi, "Drag reduction with a sliding wall in flow over a circular cylinder," AIAA 
J., 38, 715 (2000).

9 D. Barrett, M. Triantafyllou, D. Yue, M. Grosenbaugh, and M.J. Wolfgang, "Drag reduction in fish-like locomotion," J. Fluid Mech., 392, 183 (1999).

10 S. Ho, H. Nassef, N. Pornsinsirirak, Y.-C. Tai, and C.-H. Ho, "Unsteady aerodynamics and flow control for flapping wing flyers," Progress in Aerospace Sciences, 39, 635 (2003). 
List of tables

TABLE 1. Evolution of the Power Saving Ratio versus $\theta_{c}$. 
TABLE I:

\begin{tabular}{lcccccc}
\hline \hline$\theta_{c}$ & $30^{\circ}$ & $60^{\circ}$ & $90^{\circ}$ & $120^{\circ}$ & $150^{\circ}$ & $180^{\circ}$ \\
\hline $\mathrm{PSR} \times 100$ & 1.3 & 9.6 & 22.3 & 24.1 & 16.2 & 6.6 \\
\hline \hline
\end{tabular}

Bergmann, Cordier and Brancher, Physics of Fluids 


\section{List of figures}

FIG. 1. Sketch of the controlled flow configuration.

FIG. 2. Drag coefficient distribution on the cylinder surface for the uncontrolled flow.

$-\mathcal{C}_{D}, \cdots \mathcal{C}_{D}^{\nu},-\cdot-\mathcal{C}_{D}^{p}$

FIG. 3. Drag coefficient distribution on the cylinder surface for the controlled flow $\left(\theta_{c}=180^{\circ}\right.$ and $\left.\gamma=\gamma_{\text {min }}\right) . \longrightarrow \mathcal{C}_{D}, \cdots \mathcal{C}_{D}^{\nu},-\cdot-\mathcal{C}_{D}^{p}$.

FIG. 4. Variation of the mean drag coefficient with respect to the control angle $\theta_{c}$. partial control $\left(\gamma=\gamma_{\text {min }}\right) ;---$ : basic flow $(\gamma=0)$.

FIG. 5. Drag coefficient distribution on the cylinder surface for the controlled flow $\left(\theta_{c}=120^{\circ}\right.$ and $\left.\gamma=\gamma_{\text {min }}\right) . \longrightarrow \mathcal{C}_{D}, \cdots \mathcal{C}_{D}^{\nu},-\cdot-\mathcal{C}_{D}^{p}$

FIG. 6. Time evolution of the drag coefficient for the partial controlled flow $(\gamma(t)=$ $\gamma_{\min }(t)$ and $\left.\theta_{c}=120^{\circ}\right)$.

FIG. 7. Iso-vorticity contours of the partial controlled wake flow $\left(\gamma(t)=\gamma_{\text {min }}(t)\right.$ and $\theta_{c}=120^{\circ}$ ) at $t=100 .-\omega_{z}>0,---\omega_{z}<0$. (a) Organization of the near wake. (b) Whole wake. 


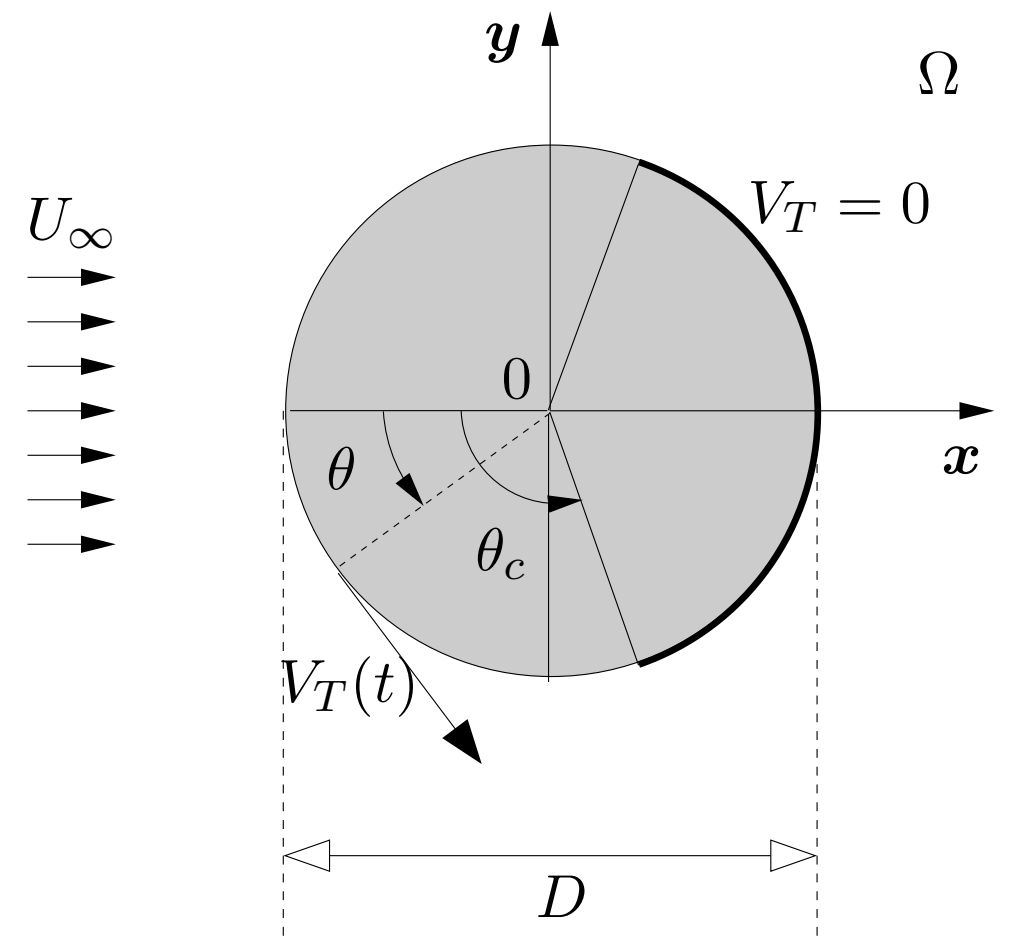

FIG. 1:

Bergmann, Cordier and Brancher, Physics of Fluids 


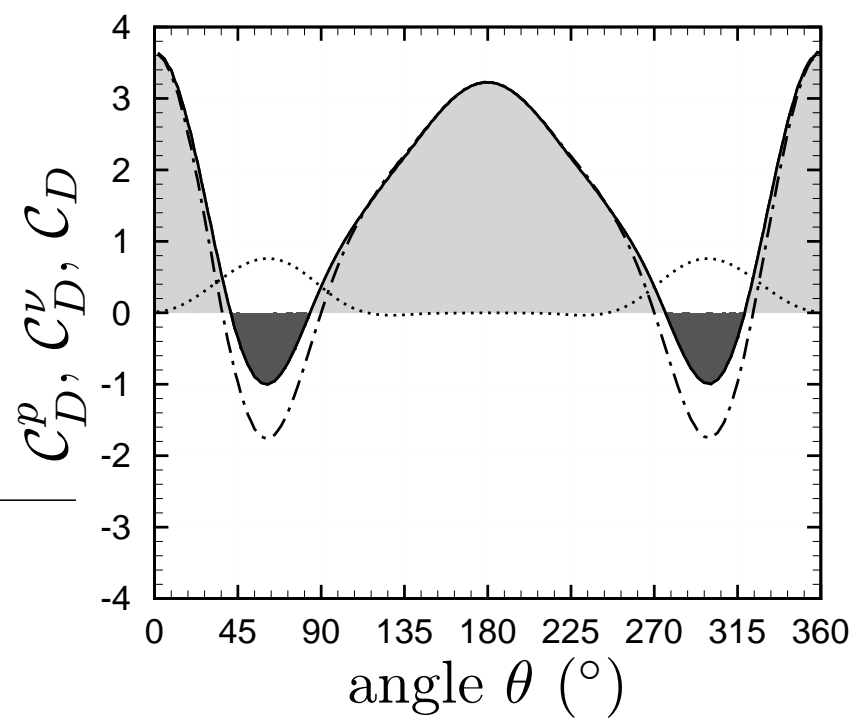

FIG. 2:

Bergmann, Cordier and Brancher, Physics of Fluids 


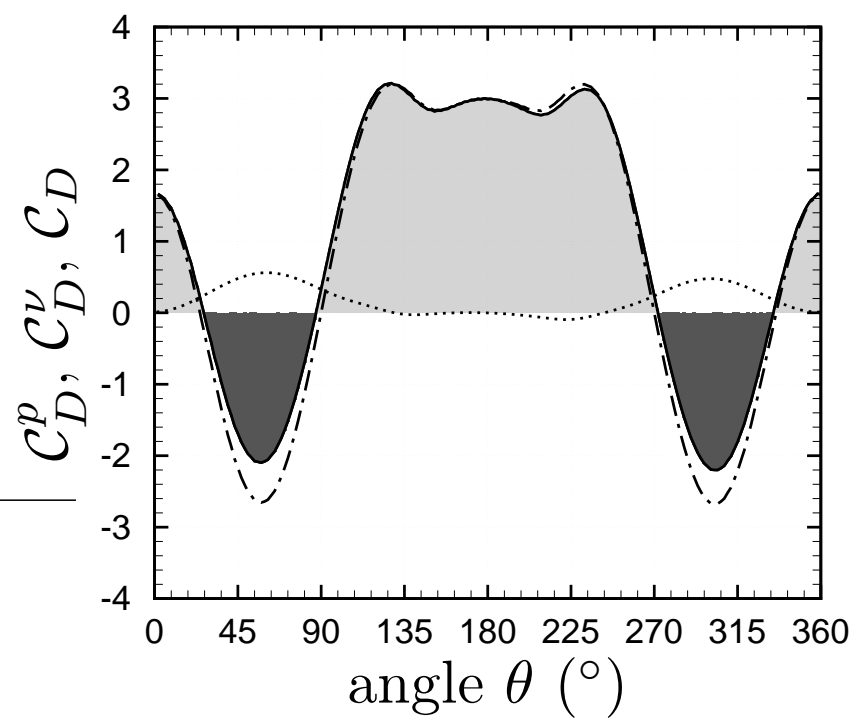

FIG. 3:

Bergmann, Cordier and Brancher, Physics of Fluids 


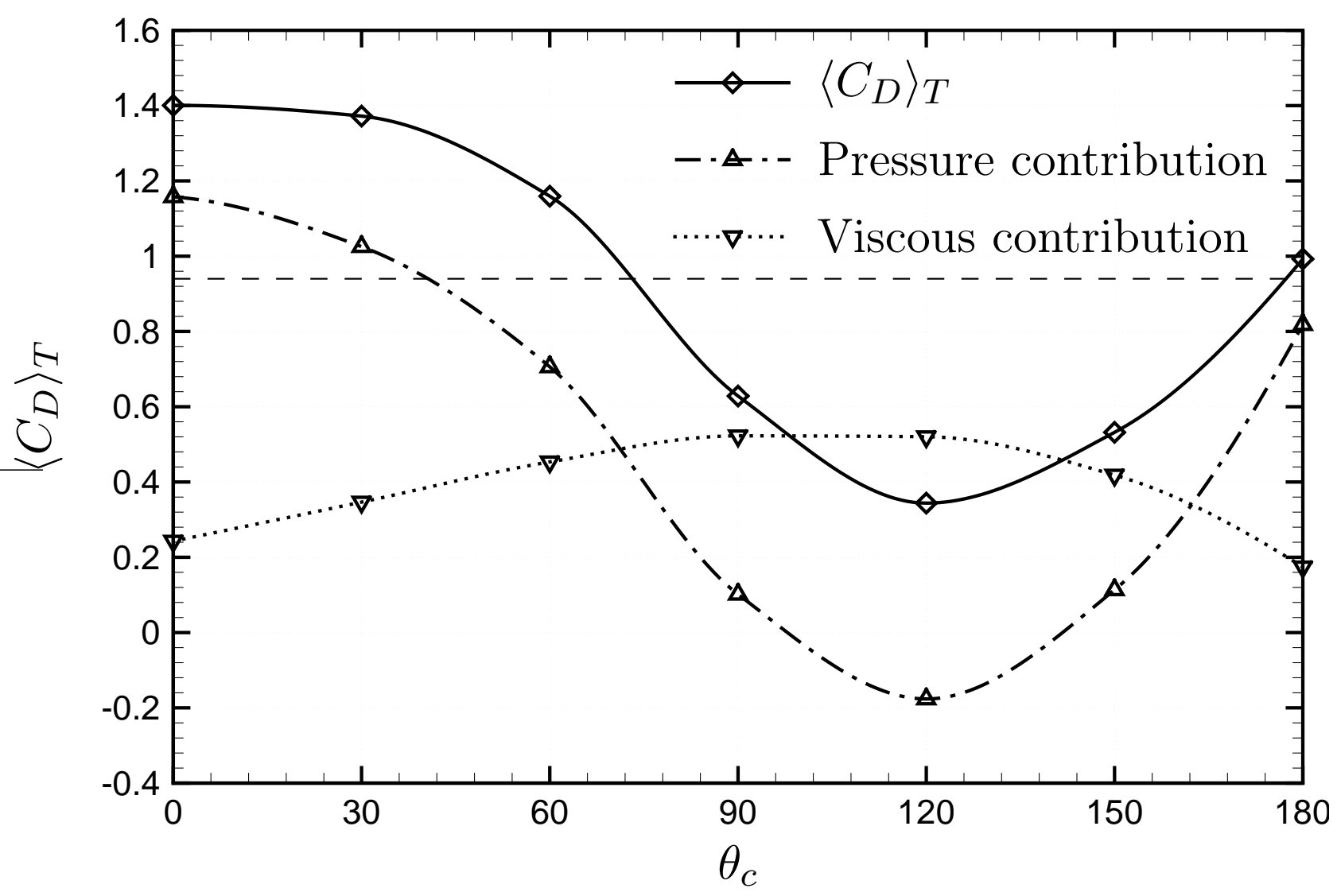

FIG. 4:

Bergmann, Cordier and Brancher, Physics of Fluids 


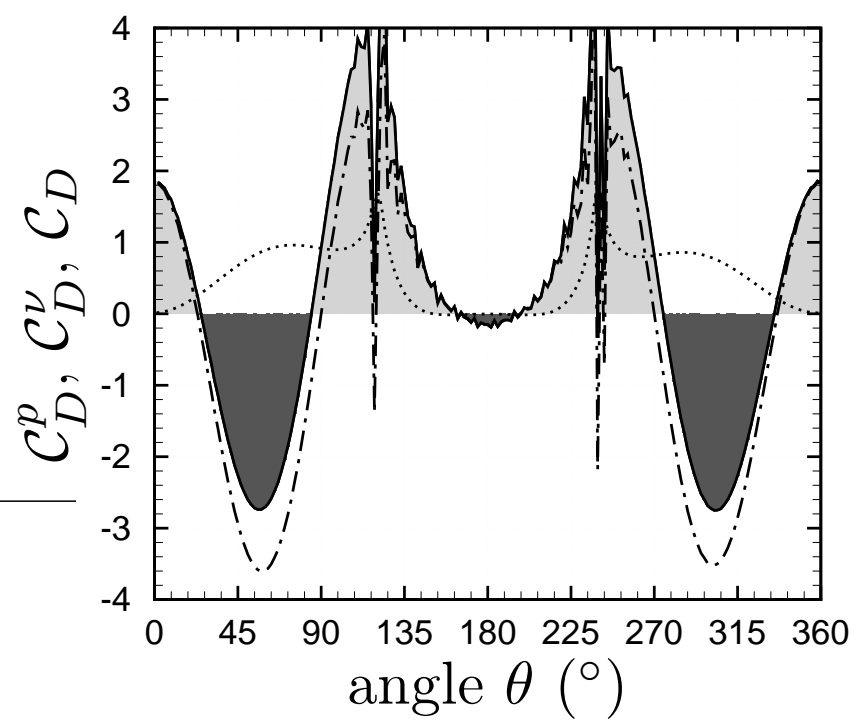

FIG. 5:

Bergmann, Cordier and Brancher, Physics of Fluids 


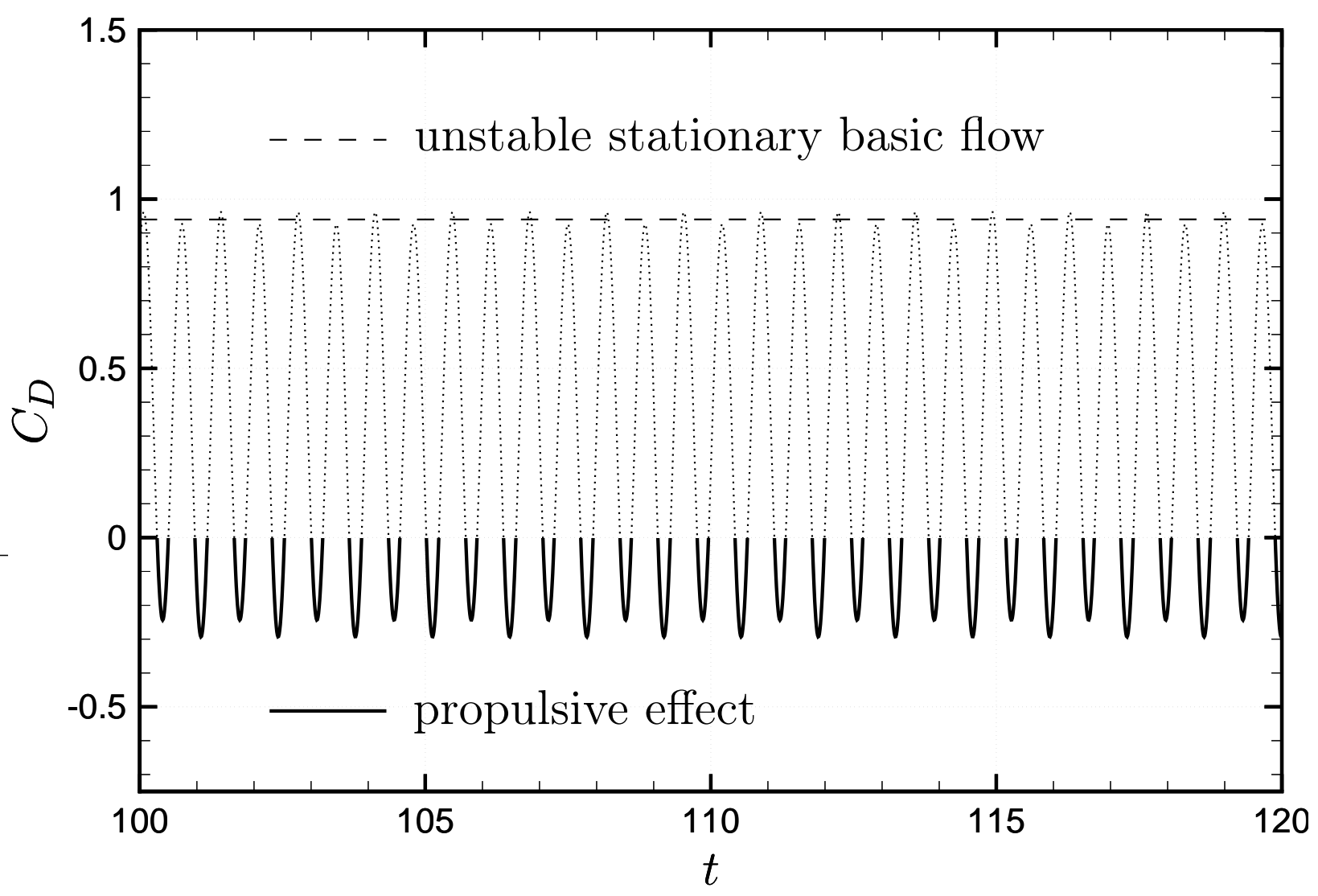

FIG. 6:

Bergmann, Cordier and Brancher, Physics of Fluids 


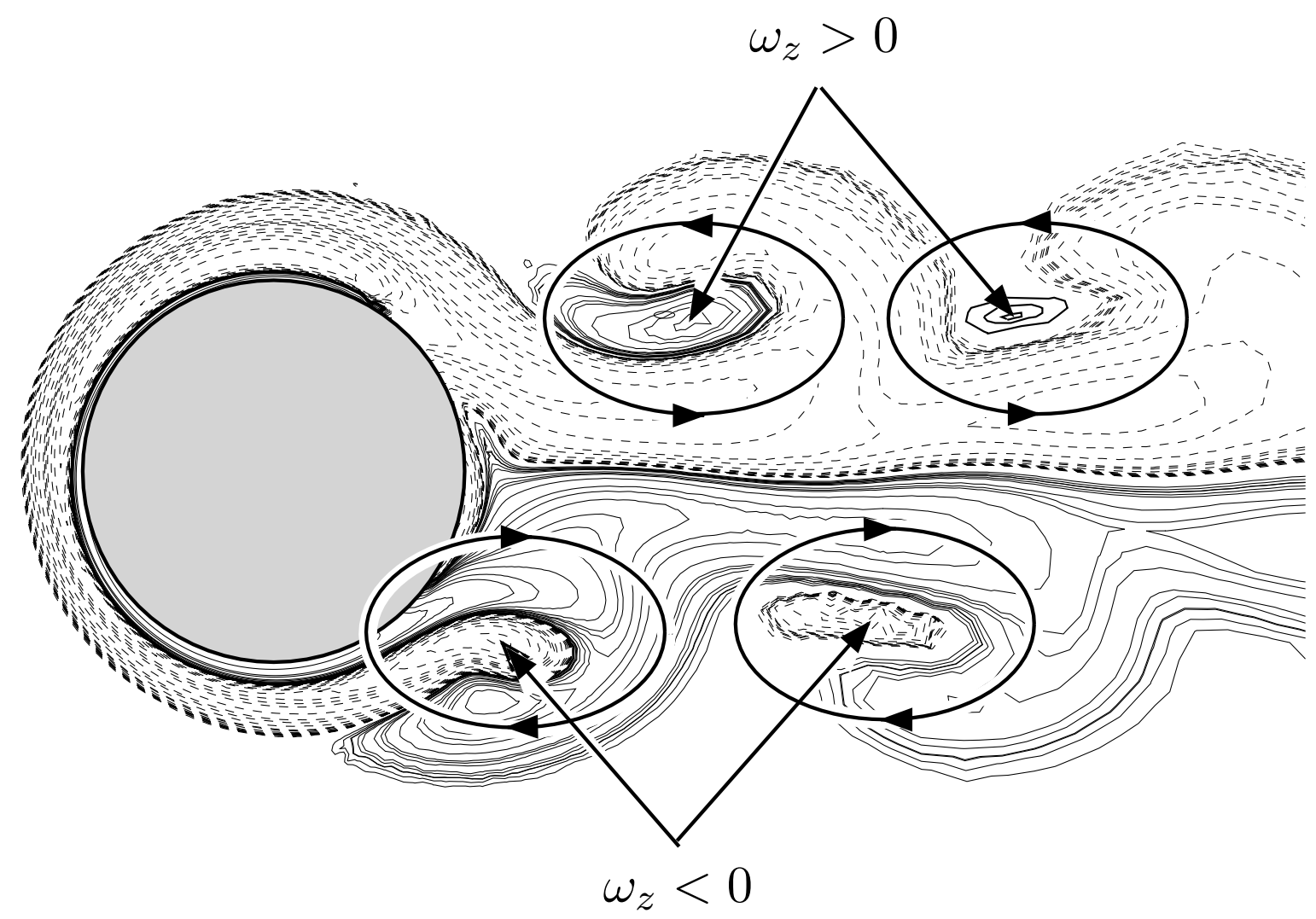

(a) Organization of the near wake.

(b)Whole wake.

FIG. 7:

Bergmann, Cordier and Brancher, Physics of Fluids 